\title{
The EFFeCt of Physical BASEd Features For RECOGNITION OF RECAPTURED IMAGES
}

\author{
S. A. A. H. Samaraweera and B. Mayurathan \\ Department of Computer Science, University of Jaffna, Sri Lanka
}

\begin{abstract}
It is very simple and easier to recapture a high quality images from LCD screens with the development of multimedia technology and digital devices. In authentication, the use of such recaptured images can be very dangerous. So, it is very important to recognize the recaptured images in order to increase authenticity. Even though, there are a number of features that have been proposed in various state-of-theart visual recognition tasks, but it is still difficult to decide which feature or combination of features have more significant impact on this task. In this paper an image recapture detection method based on set of physical based features including texture, HSV colour and blurriness is proposed. Also, this paper evaluates the performance of different distinctive featuresin the context of recognition of recaptured images. Several experimental setups have been conducted in order to demonstrate the performance of the proposed method. In all these experimental results, the proposed method is efficient with good recognition rate. Among the combination of low-level features, CS-LBP detection is to operator which is used to extract the texture feature is the most robust feature.
\end{abstract}

\section{KEYWORDS}

Image Recapture Detection, Texture, HSV, Blurriness \& CS-LBP

\section{INTRODUCTION}

The Information Technology is increasing rapidly from last century. The paper documents are replaced by digital documents. However, a photograph implies truthfulness. This technology enables digital documents to be easily modified and converted which makes our life easier in digital matters. Even though a text is a communication medium, an image accomplishes an effective communication channel for humans. Hence, in today's world a major challenge is maintenance of the trustfulness of digital images. A recaptured image is different from the common photograph in that what being captured is an image reproduction surface instead of a general scene. The Image recapture detection technique distinguishes real images from recaptured images. i.e.) images from media that displays real-scene images such as printed pictures or LCD display. Figure 1 describes the difficulties of recognizing recaptured images. Here (a) and (b) are real images, (c) and (d) are recaptured images. For an artificial system, recognizing recaptured images from real ones is an extremely complicated task.

In recent years, considerable amount of researches are conducting for image recaptured detection to restore the trustworthiness of digital images [1], [2], [3]. Restoring the intrinsic image regularities automatically and removing some common tampering anomalies automatically are several possibilities of using the image recapturing process. An important task for the current image forensic system is the recognition of the recaptured images. In other hand, an image forensic system can detect rebroadcast attacks on a biometric identification system. Therefore it is studied the problem of recaptured image detection as an application in image forensics. 
Apart from that, faked identity through recapturing of a faked print face photo has become a big issue. This problem can be identified in face authentication systems that are designed with aliveness detection for verifying a live face on mobile devices such as laptop computers and smart phones.

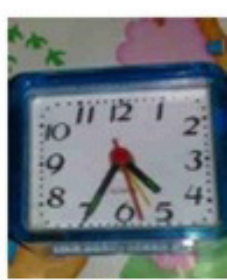

(a)

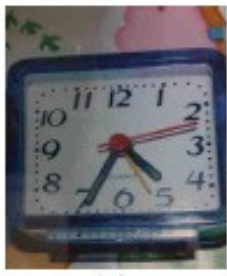

(c)

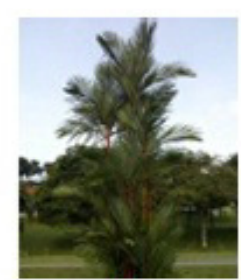

(b)

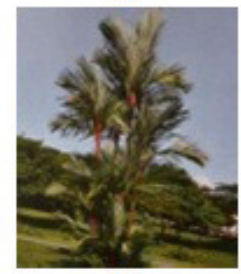

(d)

Figure 1. Difficulties of recognizing recaptured images

Image recapture detection is very useful in robot vision where differentiating the objects on a poster from the real ones is more intelligence. Another important application for IRD is in composite image detection. One way to cover composition in composite image is to recapture it.

The process of producing the real scene images and the corresponding recaptured images are shown in Figure 2. As shown in Figure 2. (a) the real image can be obtained through any type of camera. Initially the real image is captured by the any type of camera for the reproduction process. Then it is reproduced using different types of printing or display media such as printed on an office A4 paper using a colour laser printer or displayed on a LCD screen of a PC etc. Finally, the recaptured image is obtained through the camera.

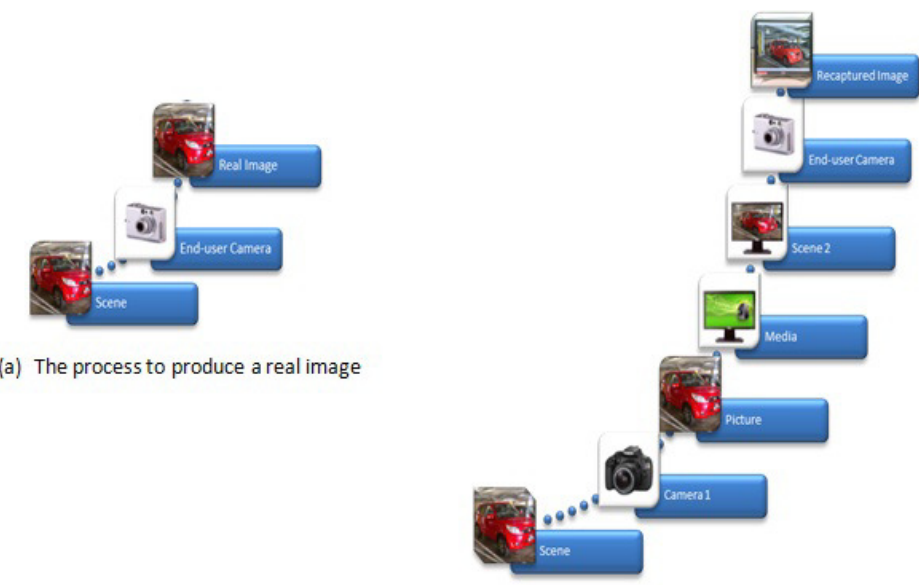

(b) The process to produce a recaptured image

Figure 2. The process of producing real image and recaptured image 
Displaying or printing a scene on any type of physical media, lead to poor quality of recaptured image. It may be identified some artefacts like texture pattern, colour fading etc. very easily. As shown in Figure 3.the low-quality recaptured images can be easily identified by the human eyes.

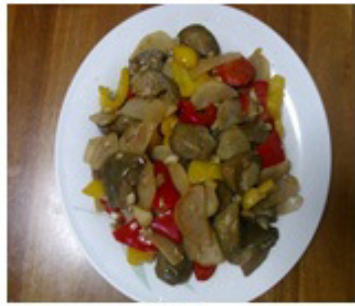

(a)

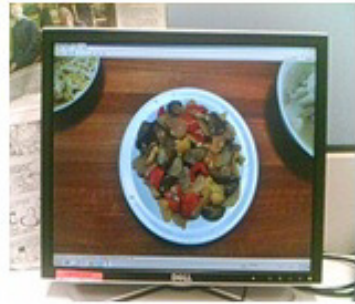

(b)

Figure 3. Comparison of a real image (a) and a recaptured image (b)

Let's discuss this by an example. Consider the displaying on LCD screen as the reproduction process as illustrated in Figure 4.Real images and corresponding recaptured images are compared with a large number of controllable settings including camera settings, LCD settings and environmental settings by Cao and Kot [5]. They concluded that visual quality of these finely recaptured images is significantly better than the casually recaptured images. So, this is a big opportunity for forgers to recapture the artificially generated scenery and use the recaptured image to fool image forensic system. Recently, a Vietnamese security group found that most commercial laptop computers with face authentication system can be easily attacked by just presenting a human face printed on an A4-size paper [6].

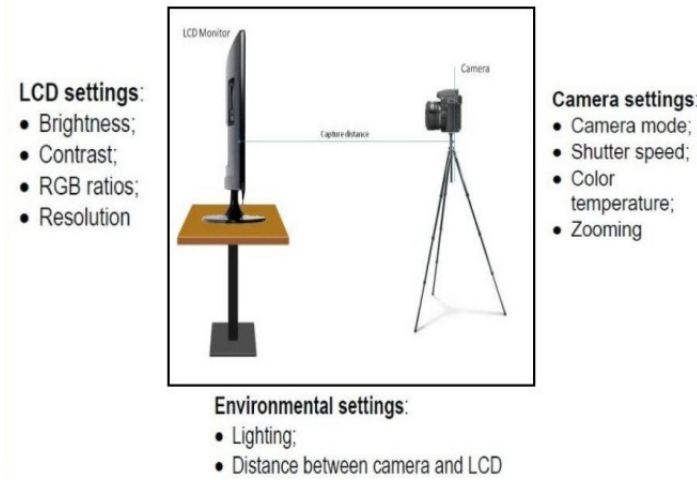

Figure 4. Some controllable settings for reproduction process on a LCD screen

The rest of this paper is organised as follows: Section II, discusses related work. Section III, describes dataset used in this paper. Section IV describes the proposed methodology for recognition of recaptured images. Section V presents the experimental results. In this section, three different experimental setups are performed in order to demonstrate the performance of proposed method. First, the proposed method is compared with several state-of-the-art methods. Second, the performances of different combinations of features used in this paper are compared. Last, the performances of brands of smart phones are compared using the proposed method as well as the most robust feature.

Finally, section VI concludes our paper. 


\section{LITERATURE REVIEW}

This section includes several approaches which are used to identify the recaptured images from real scene images as well as the studies which are related with distinguishing real scene images and recaptured images on the printing paper and LCD screens respectively.

XintingGao et al., [1] introduced a physics-based approach for recaptured image detection. The set of physics-based features is composed of the contextual background information, the spatial distribution of specularity that is related to the surface geometry, the image gradient that captures the non-linearity in the recaptured image rendering process, the colour information and contrast that is related to quality of reproduction rendering, and a blurriness measure that is related to the recapturing process. These features were used to classify the recaptured images from the real ones. This achieved significantly better classification performance on the low resolution images as compared to the wavelet statistical features.

Ke et al., [2] proposed an image recapture detection method based on multiple feature descriptors. It uses combinations of low dimensional features including texture feature, noise feature, difference of histogram feature and colour feature. The experimental result has demonstrated that this method is efficient with good detection rate of distinguishing real scene images from the recaptured ones. It possesses low time complexity.

HanyFaridy and SiweiLyu [4] presented a statistical model with first and higher order statistics which capture certain statistical regularities of natural images.

Hang Yu et al., [3] brought up a cascaded dichromatic model with the high frequency spatial variations in the specular component of a recaptured image. This distinctive feature is a result of the micro-structure on the printing paper. With a probabilistic support vector machine classifier, Cao and Kot [5] classified recaptured images on LCD screens from natural images. They perform the experiment using three types of features including texture feature using Local Binary Pattern, multi-scale wavelet statistics and colour feature.

\section{DATASET}

Bai et al., [7] found that the image resolution affects the performance of the algorithms. So, XintingGao et al., [8] presented smart phone recapture image database taken by smart phone cameras. Even though there are some publically available databases, in this work, this dataset is used due to the general resolution of the images is set to VGA $(640 \times 480)$. This dataset has constructed using following criteria.

- The images are in pair for the real image and the recaptured one taken by the same enduser camera.

- The images are consisting of outdoor natural scene, indoor office or residence scene and close-up or distant scene.

\subsection{REAL IMAGE DATASET}

The real images are obtained by any type of camera as shown in Figure 1 (a). The images in the real image dataset have produced using three popular brands of smart phones including Acer M900, Nokia N95 and HP iPAQ hw6960. These camera phones are set to auto mode whenever possible. All these three types of phones have back-facing camera. Totally I used 1094 images as real images. Table 1 lists total number of images taken from different brands of camera. 
Table 1. The number of real images.

\begin{tabular}{|l|l|}
\hline Types & Images \\
\hline Acer B & 407 \\
\hline HP B & 369 \\
\hline Nokia B & 318 \\
\hline Total & 1094 \\
\hline
\end{tabular}

\subsection{RECAPTURED IMAGE DATASET}

As illustrated in Figure 1 (b), the reproduction process is pure image-based. The images in the recaptured image dataset have produced using three types of DSLR (digital single-lens reflex) cameras including Nikon D90, Canon EOS 450D and Olympus E-520. These cameras are set to auto mode whenever possible and the resulting images have saved in JPEG format. The DSLR cameras have high resolution (greater than $3000 \times 2000$ pixels) and high quality. In constructing the recaptured dataset it has used two types of reproduction processes such as printing on a paper and displaying on a screen. The images are printed on an A4-size office paper using HP CP3505dn laser printer and Xerox Phaser 8400 ink printer. They have printed into 4R glossy and matte photos too. On the other hand, for LCD screen display they have used Dell 2007FP LCD screen (1600 x 1200 pixels). Finally the reproduced image has recaptured by the above mentioned camera phones. Table 2 lists the number of recaptured images in each reproduction process. Totally I used 1137 images as recaptured images.

Table 2. The number of recaptured images.

\begin{tabular}{|c|c|c|c|}
\hline Types of camera phones & Types & Images & Total \\
\hline \multirow{7}{*}{ Acer B } & LCD - NikonSLR & 9 & \multirow{7}{*}{269} \\
\hline & PhotoGlossy - NikonSLR & 20 & \\
\hline & PhotoGlossy - OlympusSLR & 30 & \\
\hline & PhotoMatte - NikonSLR & 30 & \\
\hline & PhotoMatte - OlympusSLR & 37 & \\
\hline & PrintInk - OlympusSLR & 50 & \\
\hline & PrintLaser - NikonSLR & 93 & \\
\hline \multirow{6}{*}{ HP B } & LCD - NikonSLR & 06 & \multirow{6}{*}{398} \\
\hline & PhotoGlossy - CannonSLR & 76 & \\
\hline & PhotoMatte - CannonSLR & 73 & \\
\hline & PrintInk - CannonSLR & 65 & \\
\hline & PrintLaser - CannonSLR & 130 & \\
\hline & PrintLaser - NikonSLR & 48 & \\
\hline \multirow{5}{*}{ Nokia B } & PhotoGlossy - OlympusSLR & 46 & \multirow{5}{*}{470} \\
\hline & PhotoMatte - OlympusSLR & 80 & \\
\hline & PrintInk - OlympusSLR & 169 & \\
\hline & PrintLaser - NikonSLR & 35 & \\
\hline & PrintLaser - OlympusSLR & 140 & \\
\hline \multicolumn{3}{|c|}{ Scenery in Total } & 1137 \\
\hline
\end{tabular}

\section{Methodology}

In this paper, an image recaptured detection method based on physical based features is proposed. A working diagram of this proposed method is illustrated in Figure 5. The images in the real image dataset and the recaptured image dataset are used for the Feature Extraction step. For each image, twenty six dimensions of features including Texture, HSV colour and Blurriness are extracted. Then to train the Support Vector Machine classifier with linear kernel, both features 
and labels are used. This is the training procedure in the proposed method. In the testing procedure, as mentioned above twenty six dimensions of features in the testing image are extracted. Then the SVM classifier with linear kernel classifies those features as the features of either a real image or a recaptured image.

\subsection{FEATURE EXTRACTION}

In general, the recaptured images and corresponding real images will never be same due to the direction of the light, distance between the camera and the scenery, sensor resolution, the lens quality and so forth. By considering this problem as a binary classification task, this paper introduces following three types of features including Texture, HSV colour and Blurriness to differentiate the recaptured images from real images.

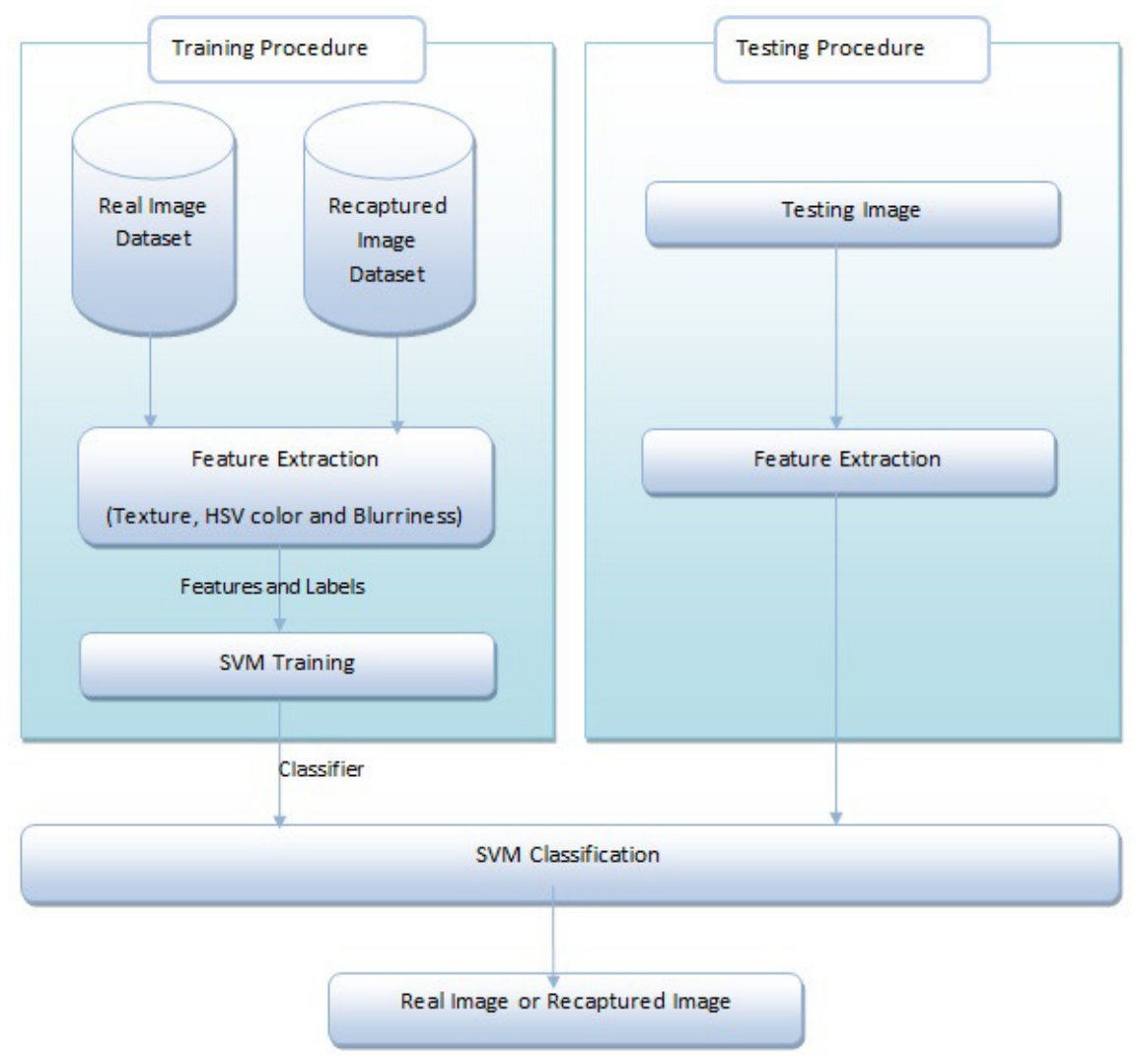

Figure 5. Diagram for the proposed image recaptured detection method

\subsubsection{TEXTURE FEATURE}

In computer vision applications, Texture plays an important role. During the past decades, so many numbers of algorithms have been presented for texture feature extraction. They can be mainly divided into two approaches: Statistical approaches and Structural approaches. Among them most commonly used algorithms are Gabor filters, Wavelet transform and so forth. Currently the local binary pattern (LBP) has received a considerable attention in many applications as a Statistical approach [9]. Due to the high dimensionality of the LBP operator, now new experiments are carrying on with the centre-symmetric local binary pattern (CS-LBP) which is an extension of LBP operator. Not only dimensionality reduction, the CS-LBP captures better the gradient information than the basic LBP. Since the CS-LBP descriptor is 
computationally simple, effective and robust for various image transformations, it is very important to present a brief review of the CS-LBP.

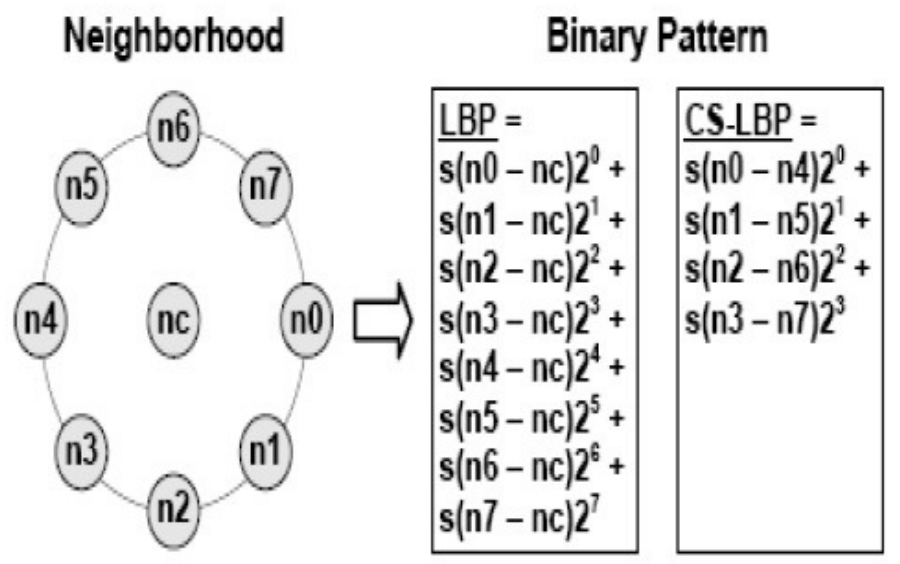

Figure 6. LBP and CS-LBP features for a neighbourhood of 8 pixels

CS-LBP operator [10] initially leads by the LBP operator. Histograms of the LBP operator are long (256) and it is not robust in flat images. CS-LBP was proposed to reduce these drawbacks.

The LBP operator compares each pixel with the centre pixel. Instead of that, the CS-LBP operator compares centre-symmetric pairs of pixels as illustrated in Figure 6. For the same number of neighbours, it produces half number of comparisons. So that the LBP produces $256\left(2^{8}\right)$ different binary patterns, whereas the CS-LBP produces only $16\left(2^{4}\right)$ different pattern for 8 neighbours. For flat areas, the operator's robustness can be increased using the gray level differences that are threshold at a small value T. Thus, the CS-LBP operator is defined by Eq. (1).

$$
C S-L B P_{R, N, T}(x, y)=\sum_{i=0}^{\frac{N}{2}-1} s\left(n_{i}-n_{i+(N / 2)}\right) \times 2^{i}, s(x)=\left\{\begin{array}{ll}
1 & \text { if } x \succ T \\
0 & \text { Otherwise }
\end{array} \quad\right. \text { Eq.(1) }
$$

Where

$(x, y)$ denotes the coordinates of a pixel,

$n_{i}$ and $n_{i+\frac{N}{2}}$ corresponds to the gray level of the center-symmetric pairs of pixels of $N$

equally spaced pixels on a circle of radius $R$.

\subsubsection{Colour Feature}

In the reproducing stage, the reproduction devices introduce some tint into the reproduced images. And also, the lighting can reduce the contrast and saturation of a recaptured image. So, the colour feature of a recaptured image looks different from its original image as shown in Figure 7. 


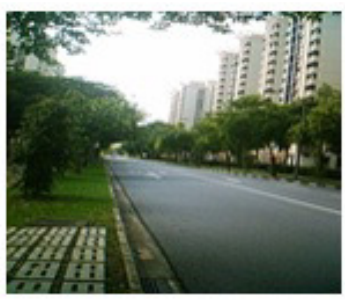

(a)

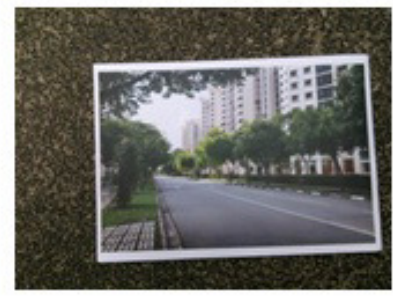

(b)

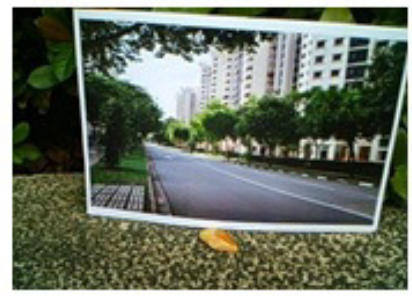

(c)

Figure 7. Comparison of the colour features introduced by the reproduction process

Colour model describes colours. Usually colour models represent a colour in the form of tuples (generally of three). The purpose of a colour model is to facilitate the specification of colours in a certain way and common standard. The RGB colour model is the most common colour model for digital images, because it retains compatibility with computer displays. However RGB has some drawbacks. RGB is non-useful for objects specification and recognition of colours. It is difficult to determine specific colour in RGB model. It reflects the use of CRTs, since it is hardware oriented system. Apart from RGB the HSV colour model is commonly used in colour image retrieval system, since HSV colours are defined easily by human perception not like RGB.

The HSV stands for the Hue, Saturation, and Value. The coordinate system is in a hexagon in Figure 8. (a). And Figure 8. (b) shows a view of the HSV colour model. The Value represents intensity of a colour, which is decoupled from the colour information in the represented image. The hue and saturation components are intimately related to the way human eye perceives colour resulting in image processing algorithms with physiological basis. As hue varies from 0 to 1.0, the corresponding colours vary from red, through yellow, green, cyan, blue, and magenta, back to red, so that there are actually red values both at 0 and 1.0. As saturation varies from 0 to 1.0 , the corresponding colours (hues) vary from unsaturated (shades of gray) to fully saturated (no white component). As value, or brightness, varies from 0 to 1.0, the corresponding colours become increasingly brighter.

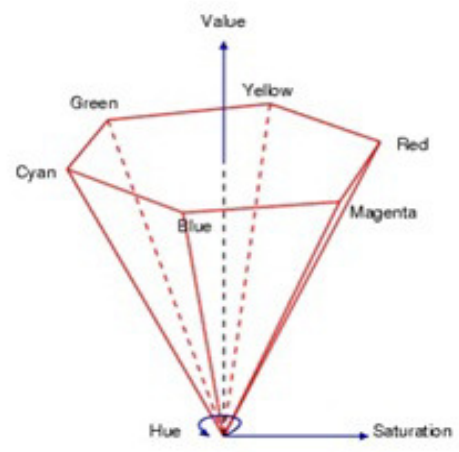

(a)

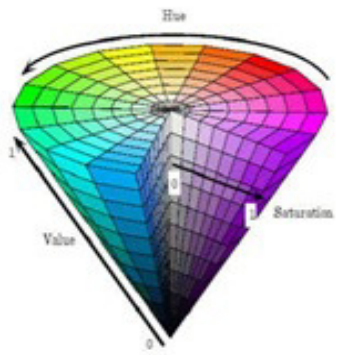

(b)

Figure 8. (a) HSV Cartesian Coordinate System (b) HSV colour model

Colour histogram and colour moments are widely used to represent the colour information of an image. Colour histogram is the approach more frequently adopted for Content Based Image Retrieval Systems. It describes the frequency of colours in images. Even though it is a widely used feature, it has some disadvantages associated with it. It is sensitive to noisy interferences. Small change in image might result in large change in histogram values and it is computationally expensive. 
Colour moments are measures that can be used to differentiate images based on their features of colour. The assumption of the basis of colour moments is that the distribution of colour in an image can be interpreted as a probability distribution. Probability distributions are characterized by a number of unique moments. For example normal distributions are differentiated by their mean and variance. Therefore it follows that if the colour in an image follows a certain probability distribution, the moments of that distribution can then be used as features to identify that image based on colour.

The mean, standard deviation and Skewness of an image are known as colour moments. In HSV colour model, a colour is defined by 3 values: Hue, Saturation, and Value. Colour moments are calculated for each of these channels in an image. An image therefore is characterized by 9 moments: 3 moments for each 3 colour channels. We will define the $i^{\text {th }}$ colour channel at the $j^{\text {th }}$ image pixel as $p_{\mathrm{ij}}$. The three colour moments can be defined as:

- Moment 1- Mean:

$$
E_{i}=\sum_{j=1}^{N} \frac{1}{N} p_{i j} \quad \text { Eq.(2) }
$$

Mean can be described as the average colour value in the image

- Moment 2- Standard Deviation:

$$
\sigma_{i}=\sqrt{\frac{1}{N} \sum_{j=1}^{N}\left(p_{i j}-E_{i}\right)^{2}} \quad \text { Eq.(3) }
$$

The standard deviation is the square root of the variance of the distribution.

- Moment 3- Skewness:

$$
s_{i}=\sqrt[3]{\frac{1}{N} \sum_{j=1}^{N}\left(p_{i j}-E_{i}\right)^{3}} \quad \text { Eq.(4) }
$$

Skewness can be described as a measure of the degree of asymmetry in the distribution. For example in HSV colour space, the variable i can take values from 1 to 3 (i.e. $1=\mathrm{H}, 2=\mathrm{S}, 3=\mathrm{V}$ ). So, the resultant feature for the image contains 9 values in the form of $3 \times 3$ matrix of the following format:

$$
\left[\begin{array}{lll}
E_{11} & E_{12} & E_{13} \\
\sigma_{11} & \sigma_{12} & \sigma_{13} \\
s_{11} & s_{11} & s_{13}
\end{array}\right]
$$

Where:

$E_{11}, E_{12}, E_{13}$ represents Mean value for HSV.

$\sigma_{11,}, \sigma_{12}, \sigma_{13}$ represents Standard deviation value for HSV.

$s_{11}, \mathrm{~S}_{12}, \mathrm{~S}_{13}$ represents Skewness value for HSV. 


\subsubsection{BLURRINESS}

In a recaptured image, there are three key factors that blurriness can arise:

- The first capture device or the printing device could be of low resolution.

- The display medium may not be in the focus range of the camera due to specific recaptured settings.

- If the end user camera has a limited depth of field, the distance background may be blurring, while the entire display medium is in focus.

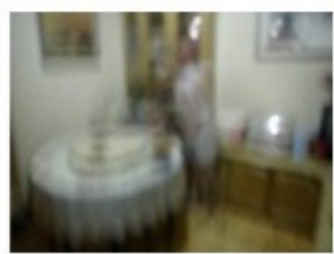

blur $_{F}=0.889063$

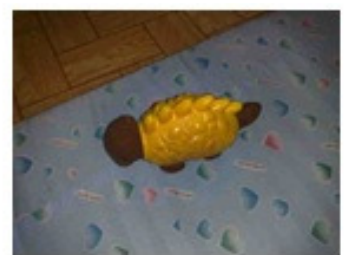

blur $_{F}=0.357930$

Figure 9. (a) and (b)

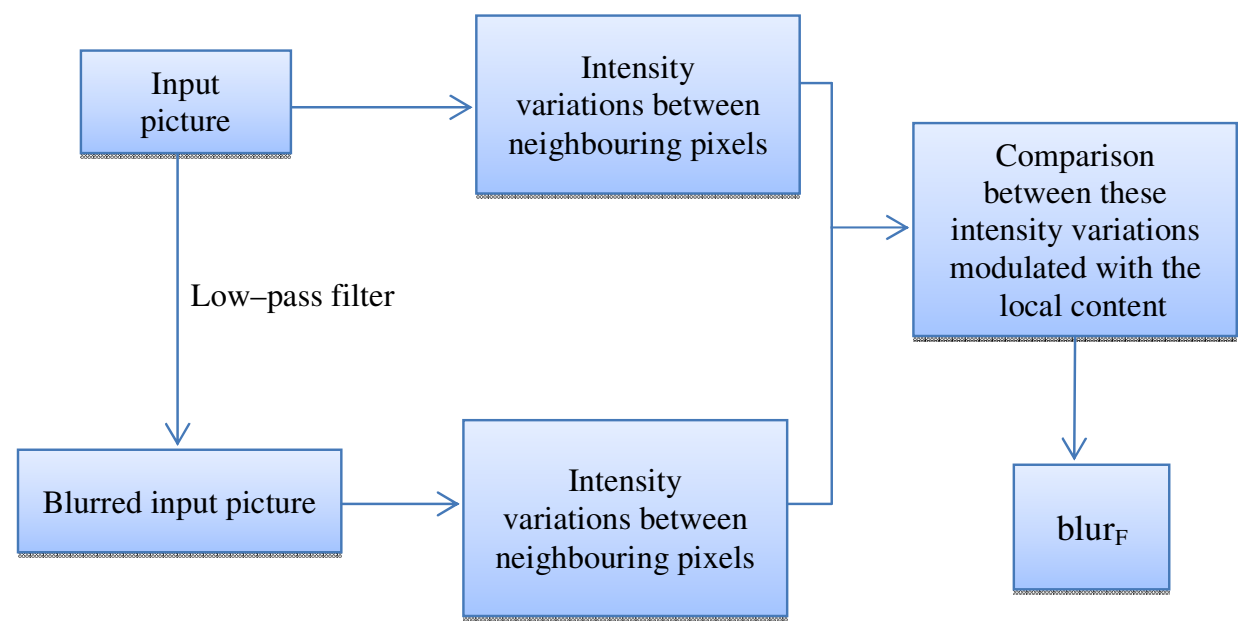

Figure 10. Simplified flow chart of the blur estimation principle

In this research work, It is explored that such information as a distinguishing feature in order to recognize whether an image is a real scene or recaptured image. The new method based on the discrimination between different levels of blur perceptible on the same image proposed by Crete et al. [11] have been used to calculate a no-reference perceptual blur metric, ranging from 0 to 1 which are respectively the best and the worst quality in term of blur perception as shown in Figure 9. (a) and (b) respectively.

As shown in Figure 10in the first step the intensity variations between neighbouring pixels of the input image is computed. Then a low-pass filter is applied and computed the variations between the neighbouring pixels. Then, the comparison between these intensity variations allows us to evaluate the blur annoyance. Thus, a high variation between the original and the blurred image means that the original image is sharp whereas a slight variation between the original and the blurred image means that the original image is already blurred. 


\subsection{Classification}

Classification consists of predicting a certain outcome based on a given input. Among various classification techniques, Support Vector Machine (SVM) is originally developed for solving binary classification problems [12].

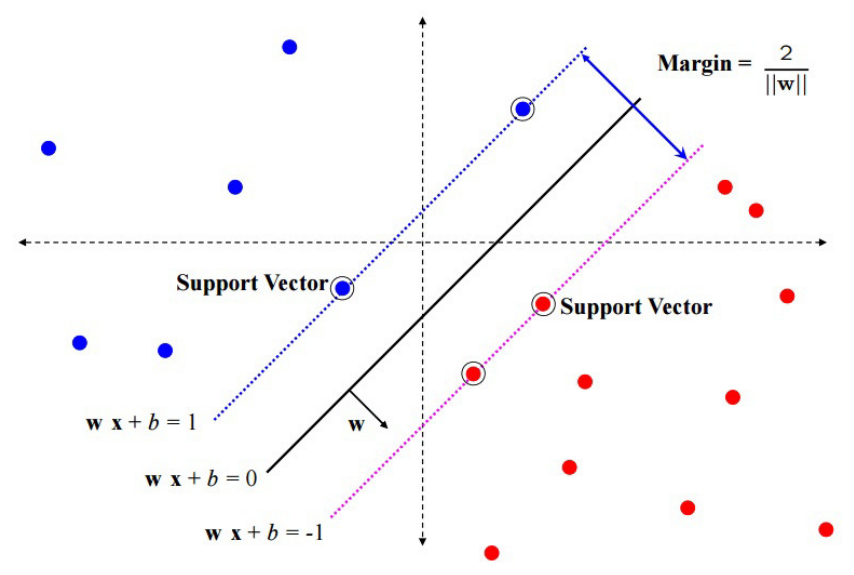

Figure 11. An example of a separable problem in a 2 dimensional space

Consider the Figure 11as an example of a linearly separable problem. Suppose it is given a set of 1 training points of the form:

$$
\left(x_{1}, y_{1}\right),\left(x_{2}, y_{2}\right), \ldots,\left(x_{i}, y_{i}\right), \ldots,\left(x_{l}, y_{l}\right) \in \Re^{n} \times \pm 1
$$

Where

$$
\begin{aligned}
& x_{i} \text { is an n-dimensional vector and } \\
& y_{i} \text { are their labels such that: } \\
& y_{i}= \begin{cases}+1 & ; \text { if the vector is classified to class }+1 \\
-1 & ; \text { if the vector is classified to class }-1\end{cases}
\end{aligned}
$$

Thus it is tried to find a classification boundary function $\mathrm{f}(\mathrm{x})=\mathrm{y}$ that not only correctly classifies the input patterns in the training data but also correctly classifies the unseen patterns. The classification boundary $\mathrm{f}(\mathrm{x})=0$, is a hyperplane defined by its normal vector $\mathrm{w}$, which basically divides the input space into the class +1 vectors on one side and the class -1 vectors on other side. Then there exists $\mathrm{f}(\mathrm{x})$ such that

$$
f(x)=w \cdot x+b, w \in \Re^{n} \text { and } \mathrm{b} \in \Re \quad \text { Eq.(5) }
$$

subject to

$$
y_{i} f\left(x_{i}\right) \geq 1 \text { for } \mathrm{i}=1,2, \ldots, \mathrm{n} \quad \text { Eq.(6) }
$$

The optimal hyperplane is defined by maximizing the distance between the hyperplane and the data points closest to the hyperplane (called support vectors). Then it is needed to maximize the margin $\mathrm{y}=2 /\|\mathrm{w}\|$ or minimize $\|\mathrm{w}\|$ subject to constraint (6). This is a quadratic programming (QP) optimization problem that can be expressed as: 


$$
\operatorname{minimize} \frac{1}{2}\|w\|^{2} \quad \text { Eq.(7) }
$$

Usually, datasets are often not linearly separable in the input space. To deal with this situation slack variables $\left(\xi_{\mathrm{i}}\right)$ are introduced into Eq. (8), where $\mathrm{C}$ is the parameter that determines the tradeoff between the maximization of the margin and minimization of the classification error. Now the QP optimization problem is given by

$$
\begin{aligned}
& \qquad \operatorname{minimize} \frac{1}{2}\|w\|^{2}+C \sum_{i} \xi_{i} \\
& \text { subject to } \\
& \qquad y_{i}\left(w \cdot x_{i}+b\right) \geq 1-\xi_{i}, \xi_{i} \geq 0 \text { for } \mathrm{i}=1,2, \ldots \quad \text { Eq.(9) }
\end{aligned}
$$

The solution to the above optimization problem has the form:

$$
f(x)=w \cdot \phi(x)+b=\sum_{i=1}^{l} C_{i} \phi\left(x_{i}\right) \cdot \phi(x)+b
$$

Where

$\Phi$ (.) is the mapping function that transforms the vectors in input space to feature space. The dot product in Eq. (10) can be computed without explicitly mapping the points into feature space by using a kernel function. Here the proposed method has used the linear kernel of the form

$$
k(x, y)=x^{T} \cdot y \quad \text { Eq.(11) }
$$

\section{EXPERIMENTAl SETUP AND TeSTING ReSUlts}

This section includes the evaluation of the proposed image recaptured detection method. In this experiment, It is used a smart phone recapture image database proposed by XintingGao et al., [8]. The real-scene images are obtained by three popular brands of smart phones including Acer M900, Nokia N95 and HP iPAQ hw6960 which have back-facing camera. The recaptured images are obtained by using three types of DSLR cameras including Nikon D90, Canon EOS 450D and Olympus E-520.

For this experiment, totally 2231 images including 1094 real-scene images and 1137 recaptured images are used. In this experimental setup, three different experimental setups are performed in order to demonstrate the performance of proposed method. First, the proposed method is compared with several state-of-the-art methods. Second, the performances of different combinations of features used in this paper are compared. Last, the performances of brands of smart phones are compared using the proposed method as well as the most robust feature.

\subsection{EXPERIMENT I}

The performance of proposed method is compared with the state-of-the-art methods. As suggested by $\mathrm{Ke}$ et al., [2], the whole dataset is partitioned into training and testing images as shown in Table 3 and measured the performance using accuracy. 
International Journal of Computer Science \& Information Technology (IJCSIT) Vol 8, No 6, December 2016

Table 3. Training and testing image selection.

\begin{tabular}{|l|l|l|}
\hline Selection & Training set & Testing set \\
\hline Selection I & $70 \%$ & $30 \%$ \\
\hline Selection II & $60 \%$ & $40 \%$ \\
\hline Selection III & $50 \%$ & $50 \%$ \\
\hline Selection IV & $30 \%$ & $70 \%$ \\
\hline
\end{tabular}

Twenty six dimensional low-level features including Texture, HSV colour and Blurriness are extracted from the training images. Table 4 shows the recognition rate of proposed method using different training and testing samples.

Table 4. Recognition rate as accuracy with different image samples on Smart Phone Recapture Image Database [8].

\begin{tabular}{|l|l|}
\hline Selection & Accuracy \\
\hline Selection I & $86.67 \%$ \\
\hline Selection II & $86.25 \%$ \\
\hline Selection III & $84.50 \%$ \\
\hline Selection IV & $78.21 \%$ \\
\hline
\end{tabular}

Table 5 compares the performance of the proposed method with state-of-the-art methods. According to the accuracy that is shown in Table 5, it can be seen that proposed method gives similar performance compared to other methods.

Table 5. Comparison of feature dimension and performance achieved.by different methods on smart phone recapture image database [8].

\begin{tabular}{|l|l|l|}
\hline Features & Dimensions & Accuracy \\
\hline Physics [1] & 166 & $91.3 \%$ \\
\hline Wavelet statistics [4] & 216 & $80.67 \%$ \\
\hline Proposed method & 26 & $86.67 \%$ \\
\hline
\end{tabular}

\subsection{EXPERIMENT II}

Table 6.Dimensions and performance on the different combinations of features.

\begin{tabular}{|l|l|l|l|l|l|}
\hline \multirow{2}{*}{ Features } & \multirow{2}{*}{ Dimensions } & \multicolumn{4}{c|}{ Accuracy } \\
\cline { 3 - 6 } & & Section I & Section II & Section III & Section IV \\
\hline Texture & 16 & $90.00 \%$ & $85.00 \%$ & $82.00 \%$ & $74.29 \%$ \\
\hline HSV & 9 & $56.67 \%$ & $62.50 \%$ & $62.00 \%$ & $54.29 \%$ \\
\hline Blurriness & 1 & $63.33 \%$ & $55.00 \%$ & $49.00 \%$ & $50.00 \%$ \\
\hline Texture + HSV & 25 & $80.00 \%$ & $75.00 \%$ & $72.00 \%$ & $70.00 \%$ \\
\hline Texture + Blurriness & 17 & $90.00 \%$ & $85.00 \%$ & $82.00 \%$ & $74.29 \%$ \\
\hline HSV + Blurriness & 10 & $70.00 \%$ & $60.00 \%$ & $58.00 \%$ & $58.57 \%$ \\
\hline
\end{tabular}

In order to find out the robust feature in recognition of recaptured images, It has computed the performance of recaptured image detection method using several sets of image features such as [texture], [HSV colour], [blurriness], [texture + HSV colour], [texture + blurriness] and [HSV colour + blurriness]. The results are shown in Table 6.

It is observed that the CS-LBP operator which is used to extract the texture feature is the most robust feature in recognition of recaptured images. 


\subsection{EXPERIMENT III}

In order to find out the smart phone which has a good quality capturing process, the proposed method is appliedwith different image samples; those are captured by three popular brands of smart phones including Acer M900, Nokia N95 and HP iPAQ hw6960. Table 7 is concluded that the proposed image recaptured detection method based on physical based features is more effective for Acer M900 than Nokia N95 and HP iPAQ hw6960.

Table 7. Performance of proposed method on the brands of smart phones.

\begin{tabular}{|l|l|l|l|l|}
\hline \multirow{2}{*}{ Brand } & \multicolumn{4}{|c|}{ Accuracy } \\
\cline { 2 - 5 } & Section I & Section II & Section III & Section IV \\
\hline Acer & $81.67 \%$ & $81.25 \%$ & $74.00 \%$ & $72.14 \%$ \\
\hline HP & $68.33 \%$ & $66.25 \%$ & $63.00 \%$ & $70.71 \%$ \\
\hline Nokia & $76.67 \%$ & $72.50 \%$ & $76.00 \%$ & $72.14 \%$ \\
\hline
\end{tabular}

According to the accuracy that is shown in Table 8, it can be seen that the CS-LBP operator which is used to extract the texture feature is more efficient for Nokia N95, HP iPAQ hw6960 and Acer M900 respectively.

Table 8. Performance of CS-LBP operator on the brands of smart phones.

\begin{tabular}{|l|l|l|l|l|}
\hline \multirow{2}{*}{ Brand } & \multicolumn{4}{|c|}{ Accuracy } \\
\cline { 2 - 5 } & Section I & Section II & Section III & Section IV \\
\hline Acer & $74.38 \%$ & $75.93 \%$ & $73.67 \%$ & $72.52 \%$ \\
\hline HP & $76.52 \%$ & $77.52 \%$ & $75.98 \%$ & $69.27 \%$ \\
\hline Nokia & $83.05 \%$ & $81.90 \%$ & $80.20 \%$ & $78.99 \%$ \\
\hline
\end{tabular}

In this experiment it is concluded that the proposed image recapture detection method has achieved a comparable classification performance on low dimensional features including texture, HSV colour and blurriness. Among them, the texture which is extracted using CS-LBP operator is crucial for the recognition problem and also the proposed method and CS-LBP operator are more effective for Acer M900 and Nokia N95 respectively.

\section{Conclusions}

In this paper, an image recapture detection method is proposed based on set of physical based features which uses combination of low-level features including texture, HSV colour and blurriness. This proposed method is efficient with good recognition rate of distinguishing realscene images from the recaptured ones. Even though the proposed method possesses low dimensional features, it works excellently in both situations where in less training as well as more training images.Among the low-level features, the texture feature plays an excellent role in order to distinguish real-scene images from the recapturedones. The CS-LBP operator which uses to extract the texture feature is significant for the recognition problem.

There is a restriction in this research work. This limitation is that the dataset is consisting with images taken only back-facing camera in three types of smart phones as described in the section Dataset. This will be an effect for the Experiment III to propose the overall performance of brands of smart phones using the proposed method.

The future work is to use the most robust feature, known as texture feature which used the CSLBP operator for extraction process, to train two dictionaries using the K-SVD approach [13]. Using these two learned dictionaries, we would be able to determine whether a given image has 
International Journal of Computer Science \& Information Technology (IJCSIT) Vol 8, No 6, December 2016

been recaptured. Another work is to extract more other features and measure the performance to find the best combinations of all the features.

\section{REFERENCES}

[1] X. Gao, T.-T. Ng, B. Qiu\&S.-F. Chang, (2010) "Single-view recaptured image detection based on physics-based features", IEEEInternational Conference on Multimedia and Expo (ICME), pp146974.

[2] Y. Ke, Q. Shan, F. Qin \& W. Min, (2013) "Image recapture detection using multiple features", International Journal of Multimedia and Ubiquitous Engineering, Vol. 8, No. 5, pp71-82.

[3] H. Yu, T. -T. Ng\&Q. Sun, (2008) "Recaptured Photo Detection Using Specularity Distribution", IEEEInternational Conference on Image Processing, pp3140-3143.

[4] H. Farid\&S. Lyu (2003) "Higher-order wavelet statistics and their application to digital forensics", IEEE Workshop on Statistical Analysis in Computer Vision.

[5] H. Cao \&A. C. Kot, (2010) "Identification of Recaptured Photographs on LCD Screens", IEEEInternational Conference on Acoustics, Speech and Signal Processing, pp1790-1793.

[6] D. Ngo, (2008) Vietnamese security firm:Your face is easy to fake, [Online], Available: http://news.cnet.com/8301-17938105-10110987.html

[7] J. Bai, T.-T. Ng, X. Gao\&Y. Q. Shi, (2010) "Is physics-based liveness detection truly possible with a single image?",IEEEInternational Symposium on Circuits and Systems (ISCAS).

[8] X. Gao, B. Qiu, J. Shen, T.-T. Ng, \&Y. Q. Shi (2011) Digital Watermarking $9^{\text {th }}$ International Workshop, IWDW Revised Selected Papers, pp90-104.

[9] W. Xiaosheng \&S. Junding, (2009) “An effective texture spectrum descriptor",Fifth International Conference on Information Assurance and Security.

[10] M. Heikkila\&C. Schmid, (2009) "Description of interest regions with Local binary patterns", Pattern Recognit., Vol. 42, No. 3, pp425-436.

[11] F. Crete, T. Dolmiere, P. Ladret\&M. Nicolas, (2007) "The blur effect: perception and estimation with a new no-reference perceptual blur metric", SPIEInternational Society for Optical Engineering.

[12] A. Ramanan, S. Suppharangsan \&M. Niranjan, (2007) "Unbalanced Decision Tree for Multi-class Classification”, IEEE International Conference on Industrial and Information Systems (ICIIS'07), pp291-294.

[13] T. Thongkamwitoon, H. Muammar \& P. L. Dragotti (2014) "Robust Image Recapture Detection using a K-SVD Learning Approach to train dictionaries of Edge Profiles", IEEE International Conference on Image Processing (ICIP), pp5317-5321.

\section{AUTHORS}

S. A. A. H. Samaraweera received the B.Sc. Special Degree in Computer Science from University of Jaffna, Sri Lanka in 2016. Her current research interests include image processing and digital image forensic.

B. Mayurathan received $\mathrm{PhD}$ Degree in Computer Science from University of Peradeniya , Sri Lanka in 2014. Her current research interests include Computer vision and Machine Learning.
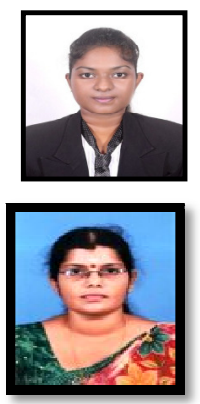